\title{
Pathological features of hypertrophic cardiomyopathy without asymmetrical septal hypertrophy
}

\author{
MASARU TANAKA, HISAYOSHI FUJIWARA, CHUICHI KAWAI \\ From the Third Division, Department of Internal Medicine, Kyoto University, fapan
}

SUMMARY In a heart with hypertrophic cardiomyopathy without asymmetrical septal hypertrophy the number of transmural myocytes, the mean size of myocytes, and the percentage area of interstitial space were similar in the ventricular septum and left ventricular posterior wall, whereas in a reported series of 14 hearts with hypertrophic cardiomyopathy with asymmetrical septal hypertrophy the number of transmural myocytes was greater in the ventricular septum than in the left ventricular posterior wall. In hearts with hypertrophic cardiomyopathy without asymmetrical septal hypertrophy the mean size of myocytes was significantly greater than that of normal hearts, but the number of transmural myocytes was not increased. The extent and distribution pattern of myocardial fibre disarray and fibrosis in the left ventricle were similar in hearts with hypertrophic myopathy whether or not asymmetrical septal hypertrophy was present.

Since the first report of "asymmetrical hypertrophy of the heart" by Teare in $1958,{ }^{1}$ asymmetrical septal hypertrophy has been accepted as one of the important clinicopathological findings in hypertrophic cardiomyopathy. ${ }^{23}$ It was reported, however, that asymmetrical septal hypertrophy is not evident in $5-30 \%$ of the cases with hypertrophic cardiomyopathy. ${ }^{45}$

Although important microscopical findings in hypertrophic cardiomyopathy, such as myocardial fibre disarray, myocardial fibrosis, and myocytic hypertrophy, have been reported in hypertrophic cardiomyopathy with asymmetrical septal hypertrophy ${ }^{6-8}$ they have not been studied in hypertrophic cardiomyopathy without septal hypertrophy. We have measured the extent of myocardial fibre disarray and fibrosis, the size of myocytes, the number of transmural myocytes, and the percentage area of interstitial space in such a heart.

\section{Case report}

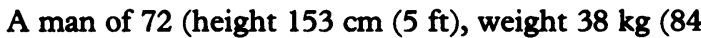
lb)) with no history of hypertension died suddenly

Requests for reprints to Dr Chuichi Kawai, The Third Division, Department of Internal Medicine, Faculty of Medicine, Kyoto University, Sakyo-Ku, Kyoto 606, Japan. during a rehabilitative admission for right hemiparesis that had been caused by a cerebral infarction five years before. He had complained of dyspnoea while in hospital, but there were no definite clinical signs of congestive heart failure. Chest $x$ ray showed slight cardiomegaly (cardiothoracic ratio $50 \%$ ) but no pulmonary congestion. The electrocardiogram showed atrial fibrillation with occasional ventricular extrasystoles (fig 1). A pansystolic murmur (Levine $3 / 6$ ) suggesting mitral regurgitation was audible at the apex. Echocardiography showed severe left ventricular concentric hypertrophy and systolic anterior movement of the anterior mitral leaflet, but asymmetrical septal hypertrophy was not seen (fig 1).

The findings at necropsy accorded with those at echocardiography. There was pronounced concentric hypertrophy of the left ventricle without asymmetrical septal hypertrophy (ventricular septum: left ventricular posterior wall, $20 \mathrm{~mm}: 18 \mathrm{~mm}=1 \cdot 1$ ) (fig 2). The heart weighed $335 \mathrm{~g}$. Important stenosis of the coronary arteries was not seen. Microscopy showed considerable hypertrophy of the myocytes, widespread myocardial fibre disarray, and diffuse interstitial fibrosis (fig 2).

We measured the extent of myocardial fibre disarray and fibrosis in a transverse slice of the left ventricle at the level of greatest hypertrophy using a general purpose colour image processor (model 


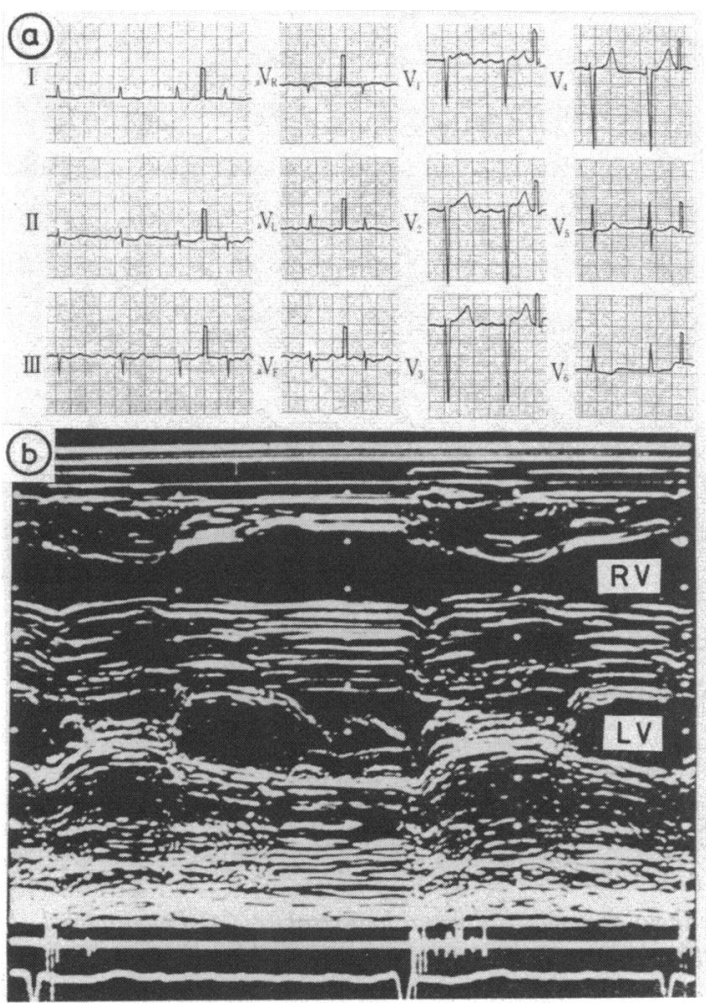

Fig 1 (a) Electrocardiogram showing atrial fibrillation. (b) $M$ mode echocardiogram showing pronounced left ventricular $(L V)$ concentric hypertrophy with systolic anterior movement of mitral valve. There is no asymmetrical septal hypertrophy. $R V$, right ventricle.

VIP-21c, Olympus), as reported elsewhere. ${ }^{7-9}$ There was disarray in $31 \%$ of the area of the ventricular septum and in $21 \%$ of the left ventricular posterior wall. The percentage areas of disarray were 27 , 38 , and $24 \%$ in the right, middle, and left third of the ventricular septum respectively and 21,15 , and $26 \%$ in the inner, middle, and outer third of the left ventricular free wall respectively. The percentage area of fibrosis was $21 \%$ in the left ventricle, $23 \%$ in the ventricular septum, and $20 \%$ in the left ventricular free wall. The percentage areas of fibrosis were 22,25 , and $21 \%$ in the right, middle, and left third of the ventricular septum respectively and 23,22 , and $17 \%$ in the inner, middle, and outer third of the left ventricular free wall respectively.

The wall thickness, number of transmural myocytes, mean size of myocytes, and percentage area of interstitial space of ventricular septum and left ventricular posterior wall were measured by the method of Fujiwara $e t a l^{6}$ on the same line, avoiding trabeculae, the papillary muscles, and the supra- ventricular crest. The transmural myocytes that we counted lay on a line transecting the ventricular septum or the left ventricular posterior wall at right angles to the endocardial surface (see table).

\section{Discussion}

The weight of the heart in the present case (335 g) was low for hypertrophic cardiomyopathy. But taking into consideration the small stature of the patient this heart was considerably hypertrophied. Moreover, other clinicopathological findings were characteristic of hypertrophic cardiomyopathy. For these reasons hypertrophic cardiomyopathy without asymmetrical septal hypertrophy was diagnosed in this case.

When we examined the extent of disarray in 14 cases of hypertrophic cardiomyopathy with asymmetrical septal hypertrophy we found that the extent of disarray was greater in the ventricular septum than in the left ventricular free wall; that the percentage area of disarray in the ventricular septum was $>30 \%$ and disarray was concentrated in the middle portion; that the percentage area of disarray in the left ventricular free wall was $>10 \%$ in 10 out of 14 cases and disarray was concentrated in the outer and middle portion. ${ }^{7}$ The extent and distribution of disarray in the present case resembled the findings in hypertrophic cardiomyopathy with asymmetrical septal hypertrophy. In hypertrophic cardiomyopathy with asymmetrical septal hypertrophy we found that the extent of fibrosis was greater in the ventricular septum than in the left ventricular free wall, in which the percentage area of fibrosis increased gradually from the outer to the inner third. ${ }^{8}$ This was also true of the present case in which there was no asymmetrical septal hypertrophy.

Some cases of hypertrophic cardiomyopathy show features of dilated cardiomyopathy at a late stage of their disease. ${ }^{1011}$ These are dilatation of the left ventricle, reduced left ventricular wall motion, and thinning of the left ventricular wall. There is also massive fibrosis, particularly in the ventricular septum, which often obliterates asymmetrical septal hypertrophy. ${ }^{1011}$ The absence of massive fibrosis excludes the possibility of the secondary disappearance of asymmetrical septal hypertrophy in the present case. Histopathological examination showed only an increase in fine interstitial fibrosis in the ventricular septum. Moreover, the left ventricular cavity was not enlarged nor was movement of the left ventricular wall reduced. These features indicate that the absence of asymmetrical septal hypertrophy in this case is not caused by thinning of the asymmetrically hypertrophied septum secondary to 


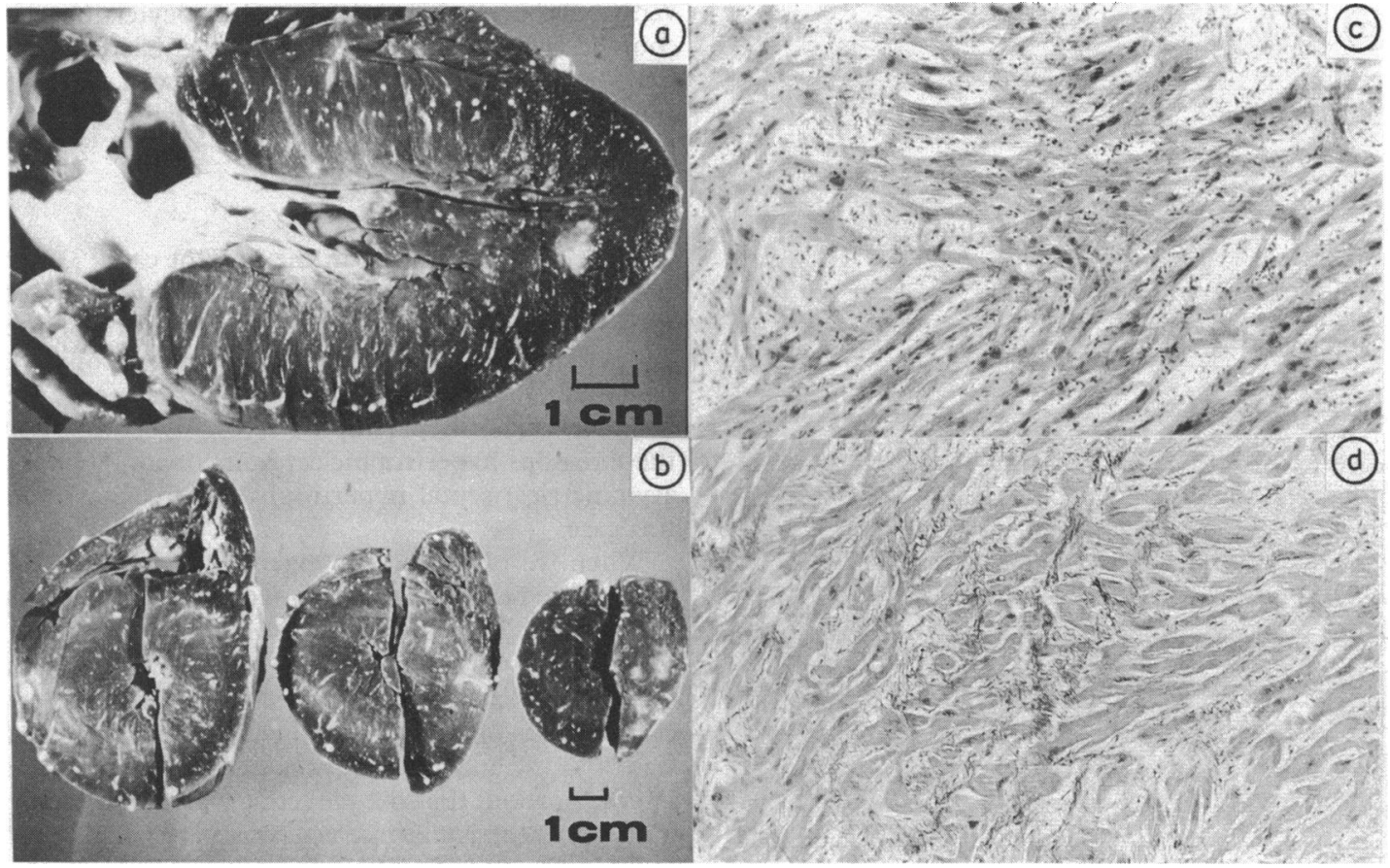

Fig 2 Pronounced concentric hypertrophy of the left ventricle without asymmetrical septal hypertrophy. (a) Longitudinal macroscopic view and (b) serial transverse views. Photomicrographs showing (c) pronounced myocardial disarray and (d) fine interstitial fibrosis.

massive fibrosis.

In our case of hypertrophic myopathy without asymmetrical septal hypertrophy there was no difference between the number of transmural myocytes, the mean size of myocytes, or the percentage area of interstitial space in the ventricular septum and in left ventricular posterior wall. In hearts from patients with hypertrophic cardiomyopathy with asymmetrical septal hypertrophy there were more transmural myocytes in the ventricular septum than in the left ventricular posterior wall and the ratio of the numbers correlated well with the ratio of wall thicknesses. ${ }^{6}$ The mean size of the myocytes and percentage area of interstitial space were similar in the ventricular septum and the left ventricular posterior wall (table). ${ }^{6}$ This suggests that the pathogenesis of symmetrical left ventricular hypertrophy in the hearts with hypertrophic cardiomyopathy without asymmetrical septal hypertrophy is associated with more even distribution of transmural myocytes in the ventricular septum and posterior wall of the left ventricle.

The size of myocytes was increased to the same extent in hearts with hypertrophic cardiomyopathy

Table Pathological features in hearts with hypertrophic cardiomyopathy (HCM) and controls

\begin{tabular}{llll}
\hline & \multicolumn{1}{l}{ VS:PW } & & Normal $^{6}$ \\
\cline { 2 - 4 } & HCM without ASH & HCM with ASH & $\frac{13(1)}{13(1)}=1 \cdot 0(0 \cdot 1)$ \\
\hline Wall thickness $(\mathrm{mm})$ & $\frac{20}{18}=1 \cdot 1$ & $\frac{25(5)}{16(2)}=1 \cdot 6(0 \cdot 2)$ & $\frac{490(60)}{480(50)}=1 \cdot 0(0 \cdot 1)$ \\
No of transmural myocytes & $\frac{530(20)}{480(20)}=1 \cdot 1$ & $\frac{630(80)}{360(70)}=1 \cdot 8(0 \cdot 3)$ & $\frac{13(1)}{14(1)}=1 \cdot 0(0 \cdot 1)$ \\
Size of myocyte $(\mu \mathrm{m})$ & $\frac{20(6)}{22(13)}=0.9$ & $\frac{19(3)}{21(2)}=0.9(0 \cdot 1)$ & $\frac{44(7)}{44(7)}=1 \cdot 0(0 \cdot 1)$ \\
Interstitial space $(\%)$ & $\frac{46(3)}{45(4)}=1.0$ & $\frac{45(4)}{43(5)}=1 \cdot 1(0 \cdot 1)$ &
\end{tabular}

ASH, asymmetrical septal hypertrophy; VS, ventricular septum; PW, posterior left ventricular wall.

Values are mean (SD). 
whether or not there was associated asymmetrical septal hypertrophy. Although the number of transmural myocytes in hearts with hypertrophic cardiomyopathy but without asymmetrical septal hypertrophy was similar to that of normal hearts, numbers were significantly increased in the ventricular septum in hypertrophic cardiomyopathy with asymmetrical septal hypertrophy. This suggests that in the asymmetrically hypertrophied septum there is both hypertrophy and hyperplasia of myocytes, whereas in the symmetrical form there is hypertrophy only.

We conclude that there are no differences in important pathological features such as myocytic hypertrophy, myocardial fibre disarray, and fibrosis between hypertrophic cardiomyopathy with and without asymmetrical septal hypertrophy and that in symmetrical hypertrophy of the left ventricle there is no disproportion in the number of transmural myocytes between the posterior wall of the left ventricle and the interventricular septum.

This project was supported in part by a research grant for cardiomyopathy from the Ministry of Health and Welfare of Japan, and by the Ministry of Education, Science and Culture of Japan.

We thank Miss $M$ Jinnai and Miss S Tomita for assistance in preparing this paper and $\mathrm{Mr} \mathrm{D}$ Mrozek for advice.

\section{References}

1 Teare D. Asymmetrical hypertrophy of the heart in young patients. $\mathrm{Br}$ Heart $\mathcal{f}$ 1958;20:1-8.

2 Henry WL, Clark CE, Epstein SE. Asymmetric septal hypertrophy: echocardiographic identification of the pathognomonic anatomic abnormality of IHSS. Circulation 1973;47:225-33.

3 Epstein SE, Henry WL, Clark CE, et al. Asymmetric septal hypertrophy. Ann Intern Med 1974;81:650-80.

4 Maron BJ, Epstein SE. Hypertrophic cardiomyopathy-recent observations regarding the specificity of three hallmarks of the disease: asymmetric septal hypertrophy, septal disorganization and systolic anterior motion of the anterior mitral leaflet. $A m \mathcal{F}$ Cardiol 1980;45:141-54.

5 Shapiro LM, McKenna WJ. Distribution of left ventricular hypertrophy in hypertrophic cardiomyopathy: a two dimensional echocardiographic study. F Am Coll Cardiol 1983;2:437-44.

6 Fujiwara H, Hoshino T, Yamana K, et al. Number and size of myocytes and amount of interstitial space in the ventricular septum and in the left ventricular free wall in hypertrophic cardiomyopathy. Am $\mathcal{F}$ Cardiol 1983;52:818-23.

7 Fujiwara H, Hoshino T, Fujiwara T, Kawai C, Hamashima $Y$. Classification and distribution of myocardial fascicle and fiber disarray in 14 hearts with hypertrophic cardiomyopathy in $25 \mu$ thick sections. Fpn Circ f 1982;46:225-34.

8 Tanaka M, Fujiwara H, Onodera T, Wu D-J, Hamashima Y, Kawai C. Quantitative analysis of myocardial fibrosis in normals, hypertensive hearts, and hypertrophic cardiomyopathy. $\mathrm{Br}$ Heart $\boldsymbol{f}$ 1986;55:575-81.

9 Fujiwara H, Kawai C, Hamashima Y. Myocardial fascicle and fiber disarray in $25 \mu$-thick sections. Circulation 1979;59:1293-8.

10 Maron BJ, Epstein SE, Roberts WC. Hypertrophic cardiomyopathy and transmural myocardial infarction without significant atherosclerosis of the extramural coronary arteries. $\mathrm{Am} \mathcal{F}$ Cardiol 1979;43:1086-102.

11 Fujiwara H, Onodera T, Tanaka M, et al. Progression from hypertrophic obstructive cardiomyopathy to typical dilated cardiomyopathy-like features in the end stage. Jpn Circ F 1984;48:1210-4. 